Pacific Journal of Mathematics

MAPPINGS OF POLYHEDRA WITH PRESCRIBED FIXED 


\title{
MAPPINGS OF POLYHEDRA WITH PRESCRIBED FIXED POINTS AND FIXED POINT INDICES
}

\author{
Helga SChirmer
}

\begin{abstract}
The following problem is studied: If points $c_{k}$ of a polyhedron and integers $i_{k}$ are given, when does there exist a selfmap within a given homotopy class which has the $c_{k}$ as its fixed points and the $i_{k}$ as its fixed point indices? Necessary and sufficient conditions for the existence of such selfmaps are established if the selfmap is a deformation and the polyhedron is of type $W$, and if the selfmap is arbitrary and the polyhedron is of type $S$. It is further shown that there always exists a selfmap of an $n$-sphere $(n \geqq 2)$ which has arbitrarily prescribed locations and indices of its fixed points. The proofs are based on Shi Gen-Hua's construction of selfmaps with a minimum number of fixed points.
\end{abstract}

1. Introduction. It is known that an arbitrarily given closed and nonempty subset of a polyhedron of type $W$ can always be the fixed point set of a suitable selfmap, and even of a deformation [2]. We now ask what happens if not only the locations of the fixed points, but also their indices are prescribed. More precisely, we deal with the following problem:

If the points $c_{k}$ of a polyhedron and the integers $i_{k}$, where $k=1,2, \cdots, m$, are given, when does there exist a selfmap within a given homotopy class which has the $c_{k}$ as its fixed points and the $i_{k}$ as its fixed point indices?

The problem is an extension of the well-known one concerning the existence of maps with a minimum number of fixed points, whose most general solution to date is due to Shi Gen-Hua [4]. We use Shi's results and methods to a considerable degree.

We first show that the number, location and indices of the fixed points of a deformation of a polyhedron can be arbitrarily prescribed with the only (obvious) condition that the sum of their fixed point indices equals the Euler characteristic of the polyhedron (Theorem 1). In the case of arbitrary selfmaps the-necessary and sufficientconditions which the fixed point indices must satisfy are naturally more complicated, and express the fact that the number and the indices of the essential fixed point classes of a map are homotopy invariant (Theorem 2). As in Shi's work [4] the assumptions which are made about the polyhedron are more restrictive in the case of 
arbitrary selfmaps than in the case of deformations, as the polyhedron has to be of type $S$ and not only of type $W$. (See $\S 2$ for definitions.)

Polyhedra of type $S$ or $W$ cannot be one-dimensional, but selfmaps with arbitrarily given fixed point sets (but not fixed point indices) were in [2] also constructed for all one-dimensional connected polyhedra. So one would also like to find selfmaps of one-dimensional polyhedra with prescribed locations and indices of their fixed point sets, but this cannot be done if only the conditions of Theorems 1 and 2 are assumed. The situation in the one-dimensional case seems to be much more complicated, and only a special case (where the one-dimensional polyhedron is acyclic and all fixed points are attractive or expulsive) has been settled so far [3].

Another open, and probably difficult, question arises if not only maps within a specific homotopy class, but all selfmaps of a polyhedron are considered. Only a very special case, namely the one where the polyhedron is a sphere, is considered here. We show that there always exists a selfmap of an $n$-sphere (where $n \geqq 2$ ) which has arbitrarily prescribed locations and indices of its fixed points.

The beginning of the paper contains two lemmas which deal with the splitting and moving of fixed points with given indices. Together with Shi's results they permit quick proofs of the later theorems. Shi's work has been included in the recent book by R. F. Brown [1], and [all references are made to this book in order to have them easily accessible. For the same reason Brown's book is used as a reference for facts about fixed point indices and fixed point classes.

2. Splitting and moving of fixed points. In this preliminary paragraph we develop, in the form of two lemmas, the tools for the proofs of the results in this paper. Some definitions are needed first.

We denote by $|K|$ a polyhedron which is the realization of a finite simplicial complex $K$, by $\sigma$ an open simplex of $|K|$, and by $\bar{\sigma}$ its closure. The carrier $\kappa(x)$ of a point $x \in|K|$ is the unique simplex for which $x \in \kappa(x)$. The star st $\sigma$ of a simplex $\sigma$ consists of all simplices which have $\sigma$ as a face. $\sigma$ is called a maximal simplex if $\sigma=$ st $\sigma$.

We use $\Phi(f)$ to denote the fixed point set of the map $f:|K| \rightarrow|K|$. The point $c \in \Phi(f)$ is an isolated fixed point if there exists an open set $U$ with $c \in U$ and $\bar{U} \cap \Phi(f)=c$. If $|K|$ is connected and if the fixed point index of $f$ on $U$, called $i(|K|, f, U)$, is defined as on 
p. 65 of [1], then it follows from the additivity axiom (see [1], p. 52) that $i(|K|, f, U)$ is independent of the choice of the open set $U$ as long as $c \in U$ and $\bar{U} \cap \Phi(f)=c$. For an isolated fixed point $c$ we therefore write $i(|K|, f, c)$ for the index of $f$ at $c$, where $i(|K|, f, c)=$ $i(|K|, f, U)$ for any such set $U$. For a point $c$ which is contained in a maximal simplex this definition coincides with the one on p. 122 of [1].

We are now ready for the two lemmas. The first states that any isolated fixed point in a maximal simplex can be split into an arbitrary number of fixed points as long as the Lefschetz number is not changed. It is a counterpart to Lemma 6 on p. 133 of [1], where fixed points are united.

LEMMA 1 (Splitting of fixed points). Let $f$ be a selfmap of the polyhedron $|K|$ and let the fixed point a of $f$ be contained in a maximal simplex $\sigma$ of dimension at least two for which $\Phi(f) \cap \bar{\sigma}=a$. Then there exists, for every set of $m$ integers $i_{1}, i_{2}, \cdots, i_{m}$ with $\sum_{k=1}^{m} i_{k}=i(|K|, f, a)$, a selfmap $f^{\prime}$ of $|K|$ which is homotopic to $f$, equals $f$ on $\mid K \| \sigma$, and has on $\sigma$ exactly $m$ fixed points $a_{k}(k=1,2$, $\cdots, m)$ with indices $i\left(|K|, f^{\prime}, a_{k}\right)=i_{k}$.

Proof. Choose an $\varepsilon>0$ for which $\bar{U}(a, 5 \varepsilon)=\{x \in|K| \mid d(a, x) \leqq$ $5 \varepsilon\} \subset \sigma$, where $d$ is the metric of $|K|$. As $f$ is continuous, there exists a $\delta>0$ with $f(\bar{U}(a, \delta)) \subset \bar{U}(a, \varepsilon)$, and we can choose it so that $0<\delta<\varepsilon$. Let $B$ denote the $p$-ball $\bar{U}(a, \delta)$, and select points $a_{1}, a_{2}, \cdots, a_{m}$ in $B \backslash a$ so that $d\left(a, a_{k}\right)$ is constant for all $k=1,2, \cdots, m$, and so that there exists a $\rho>0$ with $\delta-d\left(a, a_{k}\right)<\rho<d\left(a, a_{k}\right)$ for which the $\bar{U}\left(a_{k}, \rho\right)$ are pairwise disjoint. Put $A_{k}=B \cap \bar{U}\left(a_{k}, \rho\right)$. Then each $A_{k}$ is homeomorphic to a $p$-ball, $a \notin A_{k}$, and $A_{k}$ intersects the boundary $\partial B$ of $B$.

In order to construct a map with fixed points $a_{k}$ of index $i_{k}$ we use the notation of [1], pp. 120-121. Hence $|K|$ is imbedded into Euclidean space $R^{n}$, and

$$
h_{k}: R^{n}, \sigma, a_{k} \longrightarrow R^{n}, R^{p}, 0
$$

(which corresponds to $h_{1}$ on p. 120 of [1]) is an isometry which transforms $a_{k}$ into the origin 0 . A map $d_{k}^{\prime}: \partial A_{k} \cap \partial B \rightarrow R^{p} \backslash 0$ is defined by $d_{k}^{\prime}(y)=h_{k}(y)-h_{k} f(y)$ for all $y \in \partial A_{k} \cap \partial B$. As

$$
\begin{aligned}
\left|d_{k}^{\prime}(y)\right| & \leqq\left|h_{k}(y)\right|+\left|h_{k} \circ f(y)\right| \\
& \leqq \rho+\varepsilon+\delta<3 \varepsilon,
\end{aligned}
$$

$d_{k}^{\prime}$ is a map of the form $d_{k}^{\prime}: d A_{k} \cap \partial B \rightarrow \bar{U}(0,3 \varepsilon) \backslash 0$. 
Now take a $p$-ball $B_{k}$ with centre $a_{k}$ and with $B_{k} \subset A_{k}$, and choose generators $\bar{\mu}_{p} \in H^{p-1}\left(R^{p} \backslash 0\right)$ and $\alpha_{p} \in H^{p-1}\left(\partial B_{k}\right)$ as on p. 121 of [1]. Denote by $i: U(0,3 \varepsilon) \backslash 0 \rightarrow R^{p} \backslash 0$ the inclusion and by $\tilde{h}_{k}: \partial B_{k} \rightarrow \partial A_{k}$ the homeomorphism obtained by linear projection from the centre $a_{k}$. As $\partial A_{k} \cap \partial B$ is contractible and as $\left(\partial A_{k}, \partial A_{k} \cap \partial B\right)$ has the homotopy extension property with respect to $\bar{U}(0,3 \varepsilon) \backslash 0$, there exists an extension $d_{k}: \partial A_{k} \rightarrow \bar{U}(0,3 \varepsilon) \backslash 0$ of $d_{k}^{\prime}$ for which

$$
\tilde{h}_{k}^{*} d_{k}^{*} i^{*}\left(\bar{\mu}_{p}\right)=i_{k} \cdot \alpha_{p},
$$

where $i^{*}, d_{k}^{*}$ and $\widetilde{h}_{k}^{*}$ are the induced homomorphisms of the appropriate cohomology groups. We define a map $g_{k}^{\prime}$ on $\partial A_{k}$ by

$$
g_{k}^{\prime}(y)=h_{k}^{-1}\left(h_{k}(y)-d_{k}(y)\right) \text {. }
$$

Then

$$
\begin{aligned}
\left|h_{k} \circ g_{k}^{\prime}(y)\right| & \leqq\left|h_{k}(y)\right|+\left|d_{k}(y)\right| \\
& \leqq \delta+3 \varepsilon<4 \varepsilon
\end{aligned}
$$

therefore

$$
\begin{aligned}
d\left(a, g_{k}^{\prime}(y)\right) & \leqq d\left(a, a_{k}\right)+d\left(a_{k}, g_{k}^{\prime}(y)\right) \\
& \leqq \delta+4 \varepsilon<5 \varepsilon,
\end{aligned}
$$

and $g_{k}^{\prime}(y) \in \sigma$. As $A_{k}$ is homeomorphic to a $p$-ball, we can extend the map $g_{k}^{\prime}: \partial A_{k} \rightarrow \sigma$ linearly from $a_{k}$ to a map $g_{k}: A_{k} \rightarrow \sigma$ which has $a_{k}$ as its only fixed point. Note that then $g_{k}(y)=f(y)$ if $y \in \partial B$. We define a map $g^{\prime}: \bigcup_{k} A_{k} \cup \partial B \rightarrow \sigma$ by

$$
g^{\prime}(x)=\left\{\begin{array}{l}
g_{k}(x) \text { if } x \in A_{k}, \\
f(x) \text { if } x \in \partial B .
\end{array} \text { where } k=1,2, \cdots, m,\right.
$$

As the closure of the subset $\left(B \backslash \bigcup_{k} A_{k}\right)$ of $B$ is homeomorphic to a $p$-ball with centre $a$, and as $g^{\prime}$ is defined on its boundary, we can extend $g^{\prime}$ to a map $g: B \rightarrow \sigma$ such that $g(x)=g^{\prime}(x)$ if $x \in \bigcup_{k} A_{k} \cup \partial B$, and that $\left\{a, a_{1}, a_{2}, \cdots, a_{m}\right\}$ is its fixed point set. Then the map $f_{1}:|K| \rightarrow|K|$ given by

$$
f_{1}(x)=\left\{\begin{array}{lll}
f(x) & \text { if } & x \in|K| \mid B, \\
g(x) & \text { if } & x \in B
\end{array}\right.
$$

has the fixed point set $\left\{a, a_{1}, a_{2}, \cdots, a_{m}\right\}$ on $\sigma$, and is homotopic to $f$. It follows from the construction of $f_{1}$ with the help of the $d_{k}$, and from pp. 120-122 of [1], that $i\left(|K|, f_{1}, a_{k}\right)=i_{k}$. In consequence of the homotopy axiom (see [1], p. 52) we have

$$
i\left(|K|, f_{1}, a\right)+\sum_{k=1}^{m} i\left(|K|, f_{1}, a_{k}\right)=i(|K|, f, a),
$$


and therefore $i\left(|K|, f_{1}, a\right)=0$. Select an $\varepsilon^{\prime}$ with $0<\varepsilon^{\prime}<d\left(a, a_{k}\right)$ and $\bar{U}\left(a, 2 \varepsilon^{\prime}\right) \subset \sigma$, and a $\delta^{\prime}$ with $0<\delta^{\prime}<\varepsilon^{\prime}$ and $f_{1}\left(\bar{U}\left(a, \delta^{\prime}\right)\right) \subset \bar{U}\left(a, \varepsilon^{\prime}\right)$. According to Theorem 4 on p. 123 of [1] there exists a map $f^{\prime}:|K| \rightarrow|K|$ with $f^{\prime}(x)=f_{1}(x)$ for $x \in \mid K \| \bar{U}\left(a, \delta^{\prime}\right)$, with $d\left(f_{1}, f^{\prime}\right)<\varepsilon^{\prime}$ and which is fixed point free on $\bar{U}\left(a, \delta^{\prime}\right)$. As $f^{\prime}\left(\bar{U}\left(a, \delta^{\prime}\right)\right) \subset \bar{U}\left(a, 2 \varepsilon^{\prime}\right) \subset \sigma$, $f^{\prime}$ is homotopic to $f_{1}$ and hence to $f$. The set of fixed points of $f^{\prime}$ on $\sigma$ is $\left\{a_{1}, a_{2}, \cdots, a_{m}\right\}$ and the index $i\left(|K|, f^{\prime}, a_{k}\right)$ equals $i_{k}$.

The second lemma will show that isolated fixed points can be moved to arbitrarily prescribed points if $|K|$ satisfies a connectedness condition. More precisely we require that $|K|$ is of type $W$ ([1], p. 143), i.e. that every maximal simplex of $|K|$ is of dimension at least two and that for every two maximal simplices $\sigma, \sigma^{\prime}$ of $|K|$ there exist maximal simplices $\sigma_{1}, \sigma_{2}, \cdots, \sigma_{r}$ with $\sigma=\sigma_{1}, \sigma_{r}=\sigma^{\prime}$ and $\bar{\sigma}_{i} \cap \bar{\sigma}_{i+1}$ of dimension at least one for $i=1,2, \cdots, r-1$. Lemma 2 is an extension of Lemma 6 on p. 135 of [1], as we do not assume that $\kappa(c)$ is a maximal simplex.

Lemma 2 (Moving of fixed points). Let $|K|$ be a polyhedron of type $W$, let $f$ be a selfmap of $|K|$ with fixed point set $\Phi(f)=$ $\left\{a_{1}, a_{2}, \cdots, a_{m}\right\}$ and let $a_{1}$ be contained in a maximal simplex. Then there exists, for any $c \notin \Phi(f)$, a selfmap $f^{\prime}$ which is homotopic to $f$ and for which

$$
\Phi\left(f^{\prime}\right)=\left\{c, a_{2}, a_{3}, \cdots, a_{m}\right\} \text { and } i\left(|K|, f^{\prime}, c\right)=i\left(|K|, f, a_{1}\right) .
$$

Proof. Let $\sigma$ and $\sigma^{\prime}$ be two maximal simplices with $a_{1} \in \sigma$ and $c \in \sigma^{\prime} . \quad$ As $|K|$ is of type $W$, there exists a chain $\sigma_{1}=\sigma, \sigma_{2}, \cdots$, $\sigma_{r}=\sigma^{\prime}$ of maximal simplices such that $\bar{\sigma}_{i} \cap \bar{\sigma}_{i+1}$ has dimension at least one for $i=1,2, \cdots, r-1$. By repeated use of Lemma 6 on p. 135 of [1] we can find a map $g_{1}$ which is homotopic to $f$ and has a fixed point set $\Phi\left(g_{1}\right)=\left\{b, a_{2}, a_{3}, \cdots, a_{m}\right\}$, where $b \in \sigma^{\prime}$. We can also require that the line-segment $[b, c]$ contains no points of $\Phi(f)$, and that $g_{1}=f$ in a neighbourhood of $\left\{a_{2}, a_{3}, \cdots, a_{m}\right\}$. Next we obtain a map $g_{2}:|K| \rightarrow|K|$ by the same process (too complicated to describe briefly) which is used in the proof of Lemma 6 on p. 135 of [1] to obtain a map denoted there by $f_{2}$, and therefore arrive at a map $g_{2}$ which is homotopic to $g_{1}$, agrees with $g_{1}$ in a neighbourhood of $\left\{a_{2}, a_{3}, \cdots, a_{m}\right\}$, has the same fixed points as $g_{1}$, but has the property that $\bar{\kappa}(x) \cap \bar{\kappa}\left(g_{2}(x)\right) \neq \varnothing$ for all $x \in[b, c]$. Hence we can choose an $\eta>0$ so that the following three conditions are satisfied:

(i) $\bar{U}([b, c], \eta) \subset \mathrm{st} \kappa(c)$,

(ii) $\Phi\left(g_{2}\right) \cap \bar{U}([b, c], \eta)=b$,

(iii) $\bar{\kappa}(x) \cap \bar{\kappa}\left(g_{2}(x)\right) \neq \varnothing$ for all $x \in \bar{U}([b, c], \eta)$.

We then modify $g_{2}$ to a map $f^{\prime}$ with fixed point set $\left\{c, a_{2}, a_{3}, \cdots, a_{m}\right\}$ 
by putting $f^{\prime}(x)=g_{2}(x)$ for $x \in|K| \mid \bar{U}([b, c], \eta)$, and changing $g_{2}$ on $\bar{U}([b, c], \eta)$ by a method which is completely analogous to the one employed in the proof of Lemma 2.4 of [2]. The construction in [2] shows that $f^{\prime}$ is homotopic to $g_{2}$ and hence to $f$. As $f^{\prime}=f$ on a neighbourhood of $\left\{a_{2}, a_{3}, \cdots, a_{m}\right\}$, we have

$$
\begin{aligned}
\sum_{k=1}^{m} i\left(|K|, f, a_{k}\right) & =i\left(|K|, f^{\prime}, c\right)+\sum_{k=2}^{m} i\left(|K|, f^{\prime}, a_{k}\right) \\
& =i\left(|K|, f^{\prime}, c\right)+\sum_{k=2}^{m} i\left(|K|, f, a_{k}\right),
\end{aligned}
$$

and therefore $i\left(|K|, f^{\prime}, c\right)=i\left(|K|, f, a_{1}\right)$.

3. Mapping with prescribed fixed points and fixed point indices. We now proceed to construct deformations, and maps within an arbitrarily given homotopy class, with prescribed fixed points and indices. The method will be the same in both cases: we use Shi's [4] results to find a map with a minimum number of fixed points, and then use the splitting and the moving lemma of $\S 2$ in order to obtain a map with the prescribed fixed points.-By $\chi(K)$ we understand the Euler characteristic of $|K|$.

THEOREM 1. Let points $c_{k}$ of a polyhedron $|K|$ of type $W$ and integers $i_{k}$ be given, where $k=1,2, \cdots, m$. Then there exists a deformation which has the $c_{k}$ as its fixed points and the $i_{k}$ as its fixed point indices if and only if

$$
\sum\left\{i_{k} \mid 1 \leqq k \leqq m\right\}=\chi(K) .
$$

Proof. The necessity of the condition follows from the fact that the Lefschets number of a deformation equals the Euler characteristic of the polyhedron. (See [1], pp. 32, 52.) To prove its sufficiency, we construct a deformation $f_{1}:|K| \rightarrow|K|$ with exactly one fixed point $b$ which is contained in a maximal simplex in the same way as in the proof of Theorem 1 on p. 143 of [1] (or use Theorem 3.1 of [2]). By subdividing $K$ and using Lemma 2, if needed, we can change $f_{1}$ to a deformation $f_{2}$ with $\Phi\left(f_{2}\right)=a \in \sigma$, where $\sigma$ is a maximal simplex with $\bar{\sigma} \cap\left\{c_{1}, c_{2}, \cdots, c_{m}\right\}=\varnothing$. As $f_{2}$ is a deformation, we have $i\left(|K|, f_{2}, a\right)=\chi(K)$. Now we use Lemma 1 to construct a deformation $f_{3}$ with $\Phi\left(f_{3}\right)=\left\{a_{1}, a_{2}, \cdots, a_{m}\right\} \subset \sigma$ and $i\left(|K|, f_{3}, a_{k}\right)=i_{k}$, and then make $m$-fold use of Lemma 2 to obtain the desired deformation $f_{4}$ with fixed points $\left\{c_{1}, c_{2}, \cdots, c_{m}\right\}$ and indices $i\left(|K|, f_{4}, c_{k}\right)=i_{k}$.

In dealing with arbitrary maps rather than deformations we have to restrict the polyhedron $|K|$ further and assume that it is 
of type $S([1]$, p. 139). This means that the dimension of $|K|$ is at least three, and that the boundary of the star of each vertex of $|K|$ is connected. A polyhedron of type $S$ is always of type $W$, but the converse need not be true. (See [1], p. 143.)

The conditions on the indices in the generalization of Theorem 1 to arbitrary selfmaps will be more complicated. They require the concept of a fixed point class $F_{l}$ of a selfmap $f$ of $|K|$, and the index $i\left(F_{l}\right)$ of the fixed point class $F_{l}$, as defined in [1], pp. 86-87. The number of fixed point classes of a polyhedron is finite ([1], p. 86). A fixed point class $F_{l}$ of $f$ is called essential if $i\left(F_{l}\right) \neq 0$, and the Nielsen number $N=N(f)$ is the number of essential fixed point classes of $f$.

Both the number $N(f)$ and the indices $i\left(F_{l}\right)$ of the essential fixed point classes are homotopy invariant ([1], Chapter VI), and the conditions in Theorem 2 express precisely this fact. Theorem 2 coincides with Theorem 1 if $|K|$ is of type $S$ and $f$ is a deformation, as the Nielsen number of a deformation is 0 or 1 .

THEOREM 2. Let the selfmap $f$ of the polyhedron $|K|$ of type $S$ have the essential fixed point classes $F_{l}(l=1,2, \cdots, N)$, with indices $i\left(F_{l}\right)$, and let points $c_{k}$ of $|K|$ and integers $i_{k}(k=1,2, \cdots, m)$ be given. Then there exists a selfmap which is homotopic to $f$, has the $c_{k}$ as its fixed points and the $i_{k}$ as its fixed point indices if and only if the $c_{k}$ can be relabelled $c_{j}$, where $1 \leqq j=j(k) \leqq m$, such that either $N(f)=0$ and $\sum\left\{i_{j} \mid 1 \leqq j \leqq m\right\}=0$, or there exists a sequence of integers $m_{0}, m_{1}, \cdots, m_{N}$ with

$$
0=m_{0}<m_{1}<\cdots<m_{N}=m
$$

for which

$$
\sum\left\{i_{j} \mid m_{l-1}<j \leqq m_{l}\right\}=i\left(F_{l}\right) \text { for } l=1,2, \cdots, N .
$$

Proof. If $N(f)=0$, then Theorem 1 on p. 140 of [1] shows that there exists a map $f_{1}:|K| \rightarrow|K|$ which is homotopic to $f$ and fixed point free. In order to modify $f_{1}$ to a map with one fixed point, choose a point $a$ in a maximal simplex $\sigma$ of $|K|$, and arrange it so (by subdividing $K$, if necessary) that $\sigma \cap\left\{c_{1}, c_{2}, \cdots, c_{m}\right\}=\varnothing$. Take $\eta>0$ so that (i) $\bar{U}(a, \eta) \subset \sigma$, (ii) $f_{1}(\bar{U}(a, \eta)) \subset$ st $\kappa\left(f_{1}(a)\right)$, and (iii) $\bar{U}(a, \eta) \cap$ st $\kappa\left(f_{1}(a)\right)=\varnothing$. (Again it may be necessary to subdivide $K$ to make (iii) possible.) Let $\{\gamma(t) \mid 0 \leqq t \leqq 1\}$ be a path in $|K|$ from a to $f_{1}(a)$ for which $d(a, \gamma(t)) \neq t \eta / 2$ for all $0<t \leqq 1$. Denote, for any $x \in \bar{U}(a, \eta) \backslash a$, by $x(\eta / 2)$ and $x(\eta)$ the two unique points in which the ray from $a$ to $x$ intersects the boundaries of $\bar{U}(a, \eta)_{2}$, and $\bar{U}(a, \eta)$, 
and define a map $f_{2}:|K| \rightarrow|K|$ by

$$
f_{2}(x)= \begin{cases}f_{1}(x) & \text { if } \quad x \in K \backslash \bar{U}(a, \eta), \\ t f_{1}(x(\eta))+(1-t) f_{1}(a) & \text { if } \quad x=t x(\eta)+(1-t) x(\eta / 2), \\ \gamma(t) & \text { if } \quad x=t x(\eta / 2)+(1-t) a,\end{cases}
$$

where $0 \leqq t \leqq 1$.

It follows from the choice of $\eta$ and $\gamma(t)$ that $\Phi\left(f_{2}\right)=a$. As $f_{2}$ is homotopic to $f_{1}$, we have $N\left(f_{2}\right)=N\left(f_{1}\right)=0$ and hence $i\left(|K|, f_{2}, a\right)=0$. We now use Lemma 1 to find a map $f_{3}$ with $\Phi\left(f_{3}\right)=\left\{a_{1}, a_{2}, \cdots, a_{m}\right\} \subset \sigma$ and $i\left(|K|, f_{3}, a_{k}\right)=i_{k}$, and then Lemma 2 to find $f_{4}$ with $\Phi\left(f_{4}\right)=$ $\left\{c_{1}, c_{2}, \cdots, c_{m}\right\}$ and $i\left(|K|, f_{4}, c_{k}\right)=i_{k}$ for $k=1,2, \cdots, m$. Both $f_{3}$ and $f_{4}$ are homotopic to $f$, so that Theorem 2 holds if $N(f)=0$.

If $N(f)>0$, then Theorem 1 on p. 140 of [1], and its proof, show that there exists a map $f_{1}:|K| \rightarrow|K|$ in the homotopy class of $f$ which has $N=N(f)$ fixed points $b_{1}, b_{2}, \cdots, b_{N}$, where each $b_{l}$ is contained in a maximal simplex. Again we can, if needed, subdivide $K$ and use Lemma 2 to change $f_{1}$ to a map $f_{2}$ which is homotopic to $f$ and has fixed points $a_{1}, a_{2}, \cdots, a_{N}$, where each $a_{l}$ is contained in a maximal simplex $\sigma_{l}$ with $\bar{\sigma}_{l} \cap\left\{a_{1}, a_{2}, \cdots, a_{m}, c_{1}, c_{2}, \cdots, c_{m}\right\}=a_{l}$. We have $i\left(|K|, f_{2}, a_{l}\right)=i\left(|K|, f_{1}, b_{l}\right)=i\left(F_{l}\right)$. We now use Lemma 1 to split each $a_{l}$ on $\sigma_{l}$ into $m_{l}-m_{l-1}$ fixed points with indices $i_{j}$, for $m_{l-1}<j \leqq m_{l}$, and then Lemma 2 to move these fixed points to the prescribed locations $c_{k}$.

4. Outlook. Theorems 1 and 2 deal only with mappings within a given homotopy class. The following, much more general question arises naturally:

If points $c_{k}$, where $k=1,2, \cdots, m$, of a polyhedron $|K|$ and integers $i_{k}$ are given, when does there exist a selfmap of $|K|$ which has the $c_{k}$ as its fixed points and the $i_{k}$ as its fixed point indices?

An answer to this question with present means will be difficult to obtain, as it seems necessary to know the number of essential fixed point classes, and their indices, which can occur for the different homotopy classes of selfmaps. We restrict our attention to one very special case of the problem, namely the one where $|K|$ is a sphere. In this case no restriction on the fixed points and indices is necessary.

THEOREM 3. Let points $c_{k}$ of a sphere $S^{2}$ and integers $i_{k}$ be given, where $k=1,2, \cdots, m$. Then there exists a selfmap of $S^{2}$ which has the $c_{k}$ as its fixed points and the $i_{k}$ as its fixed point indices. 
Proof. As $S^{2}$ is not of type $S$, we cannot make use of Theorem 2. Therefore we start by giving a direct construction of a selfmap which has precisely one fixed point with index $\sum\left\{i_{k} \mid 1 \leqq k \leqq m\right\}$.

We identify the unit sphere $S^{2}$ of $R^{3}$ with the suspension of the unit circle $S^{1}=\{z \in C|| z \mid=1\}$ in the complex plane, and therefore write the points of $S^{2}$ as $\langle z, s\rangle$, with $z \in S^{1}$ and $s \in[-1,1]$. Let $d$ be the integer determined by

$$
1+d=\sum_{k=1}^{m} i_{k}
$$

and let

$$
\lambda=\lambda(s)= \begin{cases}1 / 2(1+s) & \text { if } \quad-1 \leqq s \leqq 0 \\ 1 / 2(1-s) & \text { if } \quad 0 \leqq s \leqq 1\end{cases}
$$

Then the map $f_{0}: S^{2} \rightarrow S^{2}$ defined by

$$
f_{0}(\langle z, s\rangle)=\left\langle z^{d}, s+\lambda\right\rangle
$$

is of degree $d$ and has the fixed points $u=\langle z, 1\rangle$ and $v=\langle z,-1\rangle$.

We now change $f_{0}$ to a map $f_{1}$ which is of the same degree, but has only one fixed point. For this purpose we select a point $a=\left\langle z_{0}, 0\right\rangle$ so that $a \notin\left\{c_{1}, c_{2}, \cdots, c_{m}\right\}$ and $z_{0}^{d} \neq-z_{0}$. Denote by $\gamma=\gamma(s)$, where $-1 \leqq s \leqq 1$, the unique great arc through $u, v$, and $a$, and put for $\varepsilon>0$

$$
\bar{U}(\gamma, \varepsilon)=\left\{x \in S^{2} \mid \widehat{\gamma(s) x} \leqq \varepsilon \text { for some } s \in[-1,1]\right\},
$$

where $\overparen{\gamma(s) x}$ is the distance from $\gamma(s)$ to $x$ measured along a shortest arc on $S^{2}$.

Select $\delta>0$ so that $z^{d} \neq-z_{0}$ if $\left|z-z_{0}\right| \leqq \delta$, and choose $\varepsilon=\varepsilon(\delta)>0$ so that $\langle z,-1 / 3\rangle \in \bar{U}(\gamma, \varepsilon)$ implies $\left|z-z_{0}\right|<\delta$, and also so that $\bar{U}(\gamma, \varepsilon)$ does not contain the antipode $b=\left\langle-z_{0}, 0\right\rangle$ of $a$. If $f_{0}(x)=b$ for some $x=\langle z, s\rangle \in S^{2}$, then $z^{d}=-z_{0}$ and $s=-1 / 3$, therefore $f_{0}(x) \neq b$ for all $x \in \bar{U}(\gamma, \varepsilon)$.

Any $x \in \bar{U}(\gamma, \varepsilon) \backslash a$ determines a point $y=y(x)$ as the unique point on the boundary of $\bar{U}(\gamma, \varepsilon)$ for which the shortest arc from $a$ to $y$ contains $x$. Hence we can write any $x \in \bar{U}(\gamma, \varepsilon) \backslash \alpha$ in the form $x=(1-t) a+t y$, where $0<t \leqq 1$. Let $f_{1}: S^{2} \rightarrow S^{2}$ be the map defined by

$$
f_{1}(x)= \begin{cases}f_{0}(x) & \text { if } x \in S^{2} \backslash \bar{U}(\gamma, \varepsilon), \\ (1-t) a+t f_{0}(y(x)) & \text { if } x \in \bar{U}(\gamma, \varepsilon) \backslash a, \\ a & \text { if } x=a .\end{cases}
$$


As both $f_{0}$ and $f_{1}$ map $\bar{U}(\gamma, \varepsilon)$ into $S^{2} \backslash b$, they are homotopic, and $f_{1}$ has degree $d$. The index $i\left(S^{2}, f_{1}, a\right)=i\left(S^{2}, f_{1}, S^{2}\right)$ equals the Lefschetz number of $f_{1}$ (see [1], p. 52), and hence is $1+d=\sum_{k=1}^{m} i_{k}$.

It is always possible to choose a simplicial structure of $S^{2}$ for which $a$ is contained in a maximal simplex $\sigma$ with $\bar{\sigma} \cap\left\{c_{1}, c_{2}, \cdots, c_{m}\right\}=\varnothing$. As $S^{2}$ is a polyhedron of type $W$, we can now use Lemma 1 to split $a$ into fixed points $a_{1}, a_{2}, \cdots, a_{m}$ in $\sigma$ with indices $i_{k}$, and then Lemma 2 to move each $a_{k}$ to $c_{k}$ in order to obtain the desired map.

REMARK. An $n$-sphere $S^{n}$ with $n \geqq 3$ is of type $S$, and hence an extension of Theorem 3 to such spheres is an almost immediate consequence of Theorem 2. It is easy to see that $S^{2}$ in Theorem 3 cannot, however, be replaced by $S^{1}$.

\section{REFERENCES}

1. R. F. Brown, The Lefschetz Fixed Point Theorem, Glenview, Ill., 1971.

2. H. Schirmer, Fixed point sets of polyhedra, Pacific J. Math., 52 (1974), 221-226.

3. - Attractive and expulsive fixed points on dendrites, to appear.

4. Shi Gen-Hua, On the least number of fixed points and Nielsen numbers, Chinese Math., 8 (1966), 234-243.

Received September 20, 1974 and in revised form January 22, 1975. This research was partially supported by the National Research Council of Canada (Grant A-7579).

Carleton University, Ottawa, Canada 


\title{
PACIFIC JOURNAL OF MATHEMATICS
}

\author{
EDITORS
}

\author{
RICHARD ARENS (Managing Editor) \\ University of California \\ Los Angeles, California 90024
}

\author{
R. A. Beaumont \\ University of Washington \\ Seattle, Washington 98105
}

J. DugundjI

Department of Mathematics

University of Southern California

Los Angeles, California 90007

D. Gilbarg and J. Milgram

Stanford University

Stanford, California 94305

\section{ASSOCIATE EDITORS}
E. F. BECKENBACH
B. H. NEUMANN
F. WOLF
K. Yoshida

\section{SUPPORTING INSTITUTIONS}

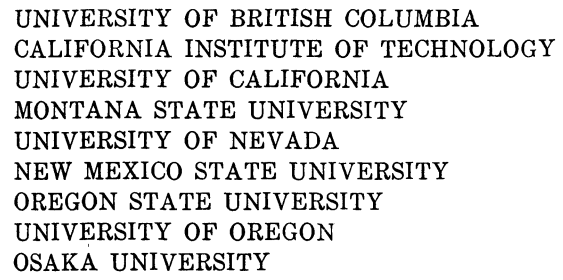

UNIVERSITY OF BRITISH COLUMBIA CALIFORNIA INSTITUTE OF TECHNOLOGY UNIVERSITY OF CALIFORNIA MONTANA STATE UNIVERSITY UNIVERSITY OF NEVADA NEW MEXICO STATE UNIVERSITY OREGON STATE UNIVERSITY UNIVERSITY OF OREGON OSAKA UNIVERSITY

\author{
UNIVERSITY OF SOUTHERN CALIFORNIA \\ STANFORD UNIVERSITY \\ UNIVERSITY OF HAWAII \\ UNIVERSITY OF TOKYO \\ UNIVERSITY OF UTAH \\ WASHINGTON STATE UNIVERSITY \\ UNIVERSITY OF WASHINGTON

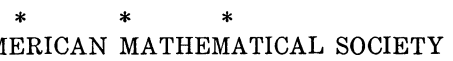

The Supporting Institutions listed above contribute to the cost of publication of this Journal, but they are not owners or publishers and have no responsibility for its content or policies.

Mathematical papers intended for publication in the Pacific Journal of Mathematics should be in typed form or offset-reproduced, (not dittoed), double spaced with large margins. Please do not use built up fractions in the text of your manuscript. You may however, use them in the displayed equations. Underline Greek letters in red, German in green, and script in blue. The first paragraph or two must be capable of being used separately as a synopsis of the entire paper. Items of the bibliography should not be cited there unless absolutely necessary, in which case they must be identified by author and Journal, rather than by item number. Manuscripts, in triplicate, may be sent to any one of the editors. Please classify according to the scheme of Math. Reviews, Index to Vol. 39. All other communications should be addressed to the managing editor, or Elaine Barth, University of California, Los Angeles, California, 90024.

The Pacific Journal of Mathematics expects the author's institution to pay page charges, and reserves the right to delay publication for nonpayment of charges in case of financial emergency.

100 reprints are provided free for each article, only if page charges have been substantially paid. Additional copies may be obtained at cost in multiples of 50 .

The Pacific Journal of Mathematics is issued monthly as of January 1966. Regular subscription rate: $\$ 72.00$ a year (6 Vols., 12 issues). Special rate: $\$ 36.00$ a year to individual members of supporting institutions.

Subscriptions, orders for back numbers, and changes of address should be sent to Pacific Journal of Mathematics, 103 Highland Boulevard, Berkeley, California, 94708.

PUBLISHED BY PACIFIC JOURNAL OF MATHEMATICS, A NON-PROFIT CORPORATION

Printed at Kokusai Bunken Insatsusha (International Academic Printing Co., Ltd.), 8-8, 3-chome, Takadanobaba, Shinjuku-ku, Tokyo 160, Japan.

Copyright (C) 1975 by Pacific Journal of Mathematics Manufactured and first issued in Japan 


\section{Pacific Journal of Mathematics}

\section{Vol. 63, No. 2 \\ April, 1976}

Joseph Anthony Ball and Arthur R. Lubin, On a class of contractive perturbations

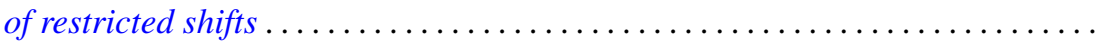

Joseph Becker and William C. Brown, On extending higher derivations generated

by cup products to the integral closure .......................

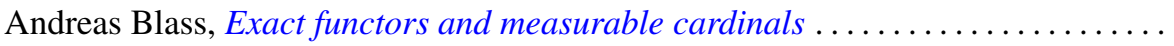

Joseph Eugene Collison, A variance property for arithmetic functions . . . . . . . . . .

Craig McCormack Cordes, Quadratic forms over nonformally real fields with a

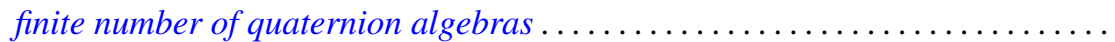

Freddy Delbaen, Weakly compact sets in $H^{1} \ldots \ldots \ldots \ldots \ldots \ldots \ldots \ldots \ldots$

G. D. Dikshit, Absolute Nörlund summability factors for Fourier series ..........

Edward Richard Fadell, Nielsen numbers as a homotopy type invariant. . ........

Josip Globevnik, Analytic extensions of vector-valued functions . . . . . . . . . . . .

Robert Gold, Genera in normal extensions . . . . . . . . . . . . . . . . . . . 389

Solomon Wolf Golomb, Formulas for the next prime

Robert L. Griess, Jr., The splitting of extensions of $S L(3,3)$ by the vector space

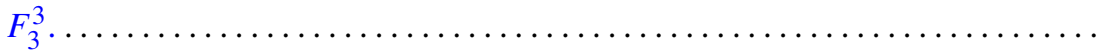

Thomas Alan Keagy, Matrix transformations and absolute summability .........

Kazuo Kishi, Analytic maps of the open unit disk onto a Gleason part.

Kwangil Koh, Jiang Luh and Mohan S. Putcha, On the associativity and commutativity of algebras over commutative rings . ..... . .

James C. Lillo, Asymptotic behavior of solutions of retarded differential difference equations.

John Alan MacBain, Local and global bifurcation from normal eigenvalues ..

Anna Maria Mantero, Sets of uniqueness and multiplicity for $L^{p}$

J. F. McClendon, Embedding metric families

L. Robbiano and Giuseppe Valla, Primary powers of a prime ideal .

Wolfgang Ruess, Generalized inductive limit topologies and barrelledness

properties.

Judith D. Sally, Bounds for numbers of generators of Cohen-Macaulay ideals

Helga Schirmer, Mappings of polyhedra with prescribed fixed points and fixed point indices.

Cho Wei Sit, Quotients of complete multipartite graphs

S. Sznajder and Zbigniew Zielezny, Solvability of convolution equations in $\mathscr{K}_{p}^{\prime}$,

$p>1$.

Mitchell Herbert Taibleson, The existence of natural field structures for finite

dimensional vector spaces over local fields

William Yslas Vélez, A characterization of completely regular fields

P. S. Venkatesan, On right unipotent semigroups ..............

Kenneth S. Williams, A rational octic reciprocity law ............

Robert Ross Wilson, Lattice orderings on the real field .......... 